\title{
Model-Based Estimation of Molten Metal Analysis in the LD Converter: Experiments at SSAB Tunnplåt $\mathrm{AB}$ in Luleå
}

\author{
Wolfgang Birk, Andreas Johansson, Alexander Medvedev, and Robert Johansson
}

\begin{abstract}
Experiences from field tests of a model-based molten metal analysis estimation system for the Linz and Donawitz converter process are reported. Experiments have been carried out during a six-month-long period on two converters at SSAB Tunnplåt $\mathrm{AB}$, Luleå, Sweden. The achieved results prove viability of the approach taken and indicate its high potential regarding estimation accuracy and robustness. It is also concluded that some further system development is necessary to enable modeling of additives and lance level before the system can be recommended for permanent installation.
\end{abstract}

Index Terms-Carbon content, estimation, implementation, nonlinear observer, steel converter, test operation.

\section{BACKGROUND}

$\mathbf{T}$ HIS PAPER is a follow-up of [1], which describes the principles behind a new model-based system for real-time estimation of carbon and silicon content in the basic oxygen steelmaking process. During 2001, extensive field tests of the system were carried out in cooperation with SSAB Tunnplåt $\mathrm{AB}$, Luleå, Sweden, and SSAB Oxelösund AB, Oxelösund, Sweden. It is well known that advanced model-based estimation techniques require extensive implementation and validation efforts before they become useful in an industrial environment. Thus, the main objective of the tests has been to evaluate estimation accuracy and robustness under realistic process conditions and collect reliable data for further system development. In this paper, the experimental results and practical experiences from a large number of converter heats at the plant in Luleå are analyzed and reported.

\section{A. LD Converter Process}

The top-blown basic oxygen method was developed 50 years ago and is employed to reduce by oxidation the contents of

Paper PID 01-38, presented at the 2001 Industry Applications Society Annual Meeting, Chicago, IL, September 30-October 5, and approved for publication in the IEEE TRANSACTIONS ON INDUSTRY APPLICATIONS by the Metal Industry Committee of the IEEE Industry Applications Society. Manuscript submitted for review October 15, 2001 and released for publication January 7, 2002.

W. Birk and A. Johansson are with the Control Engineering Group, Luleå University of Technology, SE-97187 Luleå, Sweden (e-mail: Wolfgang.Birk@sm.luth.se; Andreas.Johansson@sm.luth.se).

A. Medvedev is with the Control Engineering Group, Luleå University of Technology, SE-97187 Luleå, Sweden, and also with the Department of Systems and Control, Uppsala University, SE-75237 Uppsala, Sweden (e-mail Alexander.Medvedev@sm.luth.se).

R. Johansson is with SSAB Tunnplåt AB, SE-97188 Luleå, Sweden (e-mail: Robert.Johansson@ssab.com).

Publisher Item Identifier S 0093-9994(02)02685-3. carbon, silicon, and other contaminating components in the hot metal from the blast furnace. The converter process is run as follows. Metal scrap, hot metal, and slag-forming substances are loaded in the converter. Other additives such as ferrosilicon can be provided later in the process. Oxygen $\left(\mathrm{O}_{2}\right)$ is blown through a lance at a supersonic speed onto the metal surface and oxidizes the metal components, mainly iron (Fe), silicon ( $\mathrm{Si}$ ), manganese (Mn), and carbon (C). The oxides, together with metal droplets, form a foaming slag, in which more carbon will react with the oxides and produce carbon monoxide (CO). In combination with oxygen, some of the carbon monoxide will produce carbon dioxide $\left(\mathrm{CO}_{2}\right)$.

\section{B. Process Control}

The converter is controlled by an operator, who judges the state of the process based upon a number of measurements, e.g., a sound level measurement obtained by a sonicmeter and analysis of the off-gas [2]. The heat is completed when the content of carbon in the metal is considered to be the ordered one. The operator may also visually monitor the flame drop above the converter mouth. The quality of the final product is therefore highly dependent on the experience and the judgment of the individual operator.

A major difficulty in the way of controlling the converter process is the lack of a reliable measurement of the steel analysis. Instead, different kinds of estimation techniques are used.

An important metallurgical process variable of the converter process is the decarburization rate, i.e., the rate at which carbon is oxidized. Some of the steel mills apply simple empirical models for decarburization rate estimation based on the off-gas analysis, while others take advantage of more advanced identified dynamic models, e.g., in the form of neural networks, for different process stages and conditions. An example of a data-driven system based on a combined technique is MEFCON by Mefos [3], where both physical static mass balance and estimated dynamic process models fed by real-time measurements of the off-gas analysis and gas flows are incorporated.

At each sampling instant, MEFCON recalculates the mass balance equations of the process taking the measured off-gas analysis and flow into account. When a relatively low carbon content threshold value is hit by the estimate, a first-order dynamic model is activated to follow the decarburization process until the heat is over.

Under steady process conditions and provided accurate sensory data, this approach has been shown to produce satisfactory 
results [4]. With the perspective of fully automated closed-loop control of the converter, it is favorable to apply an estimation technique that allows analysis based on control theoretical methods. In turn, controller design and analysis, i.e., stability, performance and robustness assessment, can exploit modern control methods.

\section{ESTIMATION TECHNIQUE}

The model of the LD converter is based on a mathematical description of the physical and chemical processes taking place in the process during a heat. It is expressed as a system of two nonlinear differential equations describing the evolution of carbon and silicon content as a function of material flow of oxygen and additives. Therefore, the process model is independent of the actual converter individual it works with. Of course, this is achieved at cost of quite coarse description of the physical and chemical phenomena and should be compensated for via a robust design of an observer for the molten metal analysis estimation problem.

The purpose of the observer is to project sensory data onto a mathematical model of the process, thus separating the measurements into two parts-one comprising the data complying with the model dynamics, and another, usually called residual, including instances generated by model uncertainty and disturbances. In the observer, the process model is conventionally described by a system of differential equations. This form as well enables estimation of otherwise unmeasurable process variables. The residual is fed back to the process model in order to guarantee observer stability and enhance convergence of the estimates. This is very much similar to the prediction-correction algorithms used in numerical integration. However, the actual process dynamics are known only approximately.

An observer comprised of a nonlinear simplified mathematical model of the LD converter [2] and a nonlinear feedback is employed in order to obtain real-time estimates of the molten metal analysis. Measurements of the oxygen flow through the lance as well as the analysis and the flow of the off-gas are used for updating the process model. The observer design method is based on evaluating the sensitivity functions of the process output signals, i.e., the off-gas analysis, with respect to the process states, i.e., the carbon and silicon content [5].

\section{IMPLEMENTATION}

The field test of the model-based molten metal analysis estimation system (SafeCon) are performed at the plant in Luleå, Sweden. In order to reduce implementation time for the test series, SafeCon is installed on the same process computer as MEFCON. Thereby, SafeCon can exploit MEFCON's data acquisition routines.

Since SafeCon is not yet integrated with the control system of the converter, the correct handling of events and initialization of the algorithm is very important for the implementation.

\section{A. Process Computer}

MEFCON is installed on a Compaq Alphastation running OpenVMS as the operating system. Fig. 1 shows the structure

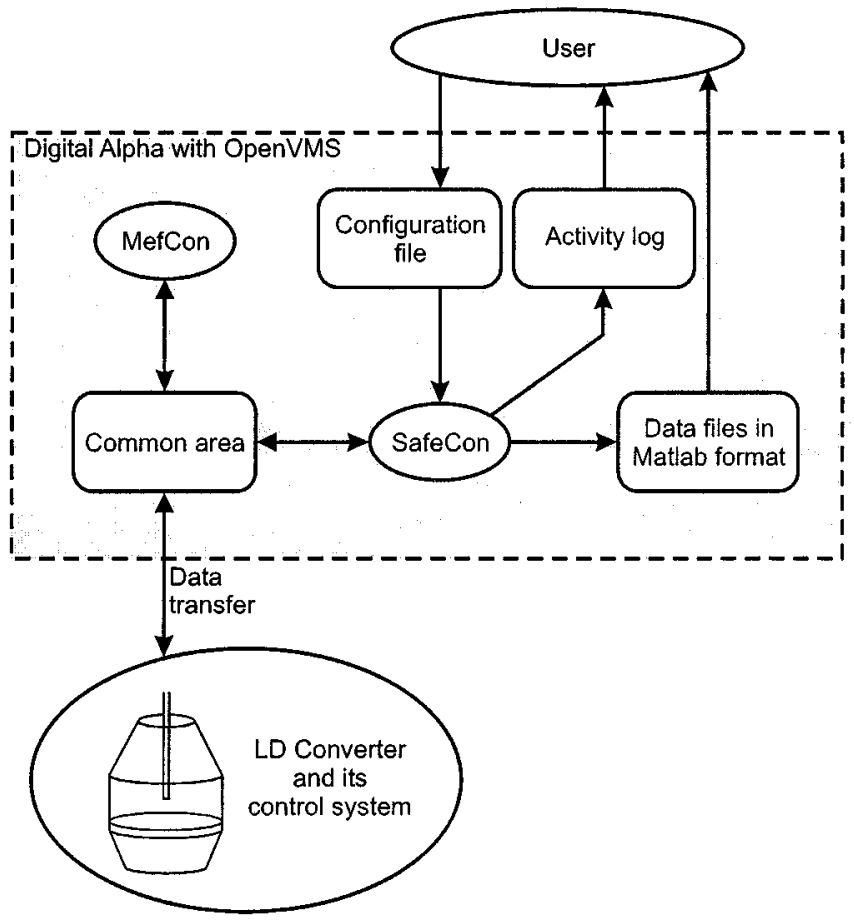

Fig. 1. Implementation of SafeCon.

of the implementation, signal flow, and how SafeCon accesses the acquired process data.

In Fig. 1, it can be seen that MEFCON and SafeCon have access to the same common area, which is updated with process data by data acquisition processes and by both MEFCON and SafeCon. The common area not only contains raw data, but it can also store preprocessed data and act as a transfer buffer between processes.

SafeCon uses the common area to acquire raw data from the process and preprocessed data from MEFCON, which are mainly flags that indicate events in the LD converter. Furthermore, the generated estimates are written back to the common area and are presented to the operators via the human-machine interface.

SafeCon itself is running as an asynchronous system process with a fixed cycle time of $1 \mathrm{~s}$. In order to give the user a minimum degree of interaction with SafeCon, a configuration file is used, which is read by SafeCon before a new heat is started. Consequently, changes in the configuration take effect without restarting SafeCon.

During a heat, SafeCon acquires external data from the LD converter process, generates internal data, i.e., process state information, and generates output data, i.e., molten metal analysis in real time. These data and additional information on the heat are written to files in Matlab format. Each heat is assigned to a separate file. The data files can then be accessed and analyzed by the user.

Moreover, SafeCon generates an activity log, which contains time-stamped information on all activities.

The data files are formatted so that a simulation environment on a PC computer can directly access the data of a heat and simulate it. Hence, debugging, redesign, and refinement of SafeCon are facilitated. 
TABLE I

EVENTS AND THEIR DEFINITIONS

\begin{tabular}{l|l}
\hline Event & Definition \\
\hline \hline Start of heat & $\begin{array}{l}\text { Lance in final position and oxygen } \\
\text { flow switched on }\end{array}$ \\
\hline End of heat & Converter tilted for tapping \\
\hline Interruption of heat & $\begin{array}{l}\text { Oxygen flow switched off and heat } \\
\text { started }\end{array}$ \\
\hline Restart of heat & $\begin{array}{l}\text { Oxygen flow switched on, lance in } \\
\text { final position and heat interrupted }\end{array}$ \\
\hline $\begin{array}{l}\text { Introduction of addi- } \\
\text { tive }\end{array}$ & $\begin{array}{l}\text { Accumulated amount for the addi- } \\
\text { tive increases }\end{array}$ \\
\hline
\end{tabular}

\section{B. Events}

Since SafeCon is implemented as a continuously running process, an event-driven state machine has to be designed that detects key events and changes the state of the estimator.

Key events are as follows:

- start of heat;

- end of heat;

- interruption of heat;

- restart of heat;

- introduction of additive.

By combining data from the common area and detecting flanks in the data, all key events can be detected. Still, some events do not occur instantaneously, whereby a definition of the time of occurrence becomes uncertain, e.g., start of heat.

When a heat is started, the oxygen is turned on when the lance is not yet in place. Consequently, decarburization starts before the final lance position is reached. Assuming that decarburization during the early beginning of a heat is negligible, this event is defined as both final lance position is reached and oxygen flow is switched on.

In case of the end of heat, the situation is not that crucial, as the estimator freezes in its current states, when the oxygen flow is switched off. Thus, a prolonged attendance of the estimator is not of any harm and the event is defined to occur when the converter is tilted for tapping.

Moreover, this definition of end of heat facilitates the detection of interruption of heat or restart of heat. An interruption often occurs in the early stages of a heat and can be caused by slopping, malfunction, or a congestion in the steel casting plant, whereas a restart is triggered when the operators have not reached the desired final carbon content.

The event introduction of additive is well defined in time and does occur abruptly. Thence, a detection from data is an elementary task.

A summary of the definitions for all events is give in Table I.

\section{Initialization}

When start of a heat is detected, SafeCon should be initialized with the initial conditions of the newly charged converter. Important contiguities are hot metal weight and content of silicon and carbon. Due to varying time delays in the metal analysis of the hot metal, these contiguities may not be available at the start, which prevents the correct initialization of SafeCon. However, because of the feedback in the estimation algorithm, deviations in the initial values do not necessarily lead to errors in the final estimate.

Thus, if the hot metal analysis is delayed, a prediction of the analysis, which is rather coarse, is used. An immediate consequence is a loss of estimation accuracy, and converter operators have to be informed. In a later version, SafeCon should enter a data-buffering mode, where the data are stored in a firstin-first-out (FIFO) buffer in real time. As soon as the analysis arrives, SafeCon continues to buffer but also spawns a nonsynchronized subprocess that simulates the heat. When, finally, all buffered data are processed, SafeCon returns from the buffering mode to the standard mode, where the estimator runs online in real time again.

Moreover, initialization must not occur after interruption of heat or before restart of heat, as it would devastate the estimation result.

\section{Restart}

When restart of heat takes place, the final carbon content in the hot metal is not close enough to the desired one. Usually, the operators initiate a new shorter heat with the same load in the converter.

SafeCon has to disregard the restart and has to freeze its estimate during the renewed heat.

\section{E. Additives}

An often-used practice of the LD converter operators is to submit additives to the metal bath in the final stage of the heat. Sometimes, additives are supplied to the process, even after the end of the heat.

Then, deviations between estimate and molten metal analysis come about. The effect of additives on the behavior of the estimate has yet to be studied.

Currently, no bounds for estimation accuracy are given. This should be provided to the operators in a later version to improve reliability.

\section{Preliminary Results}

Fig. 2 shows the results from all heats without late additives between January-April 2001 at the plant in Luleå. Included in the figure is also a normal distribution with the same standard deviation for reference. Clearly, the distribution of the estimation error is not completely random, which indicates that improvements are possible.

Note, for example, the high representation of positive errors between $0.005 \%-0.015 \%$. One possible explanation for this is due to the observer structure where the feedback is weighted by the sensitivity of the output with respect to the carbon and silicon contents. If the estimated carbon content is too high during the blow, then the feedback will be too weak, which leads to slower convergence compared to the case when the estimated carbon content is too low.

Fig. 3 shows the errors for a number of heats. There is obviously some correlation from one heat to the next. This can be utilized by predicting the error and thereby reducing it. 


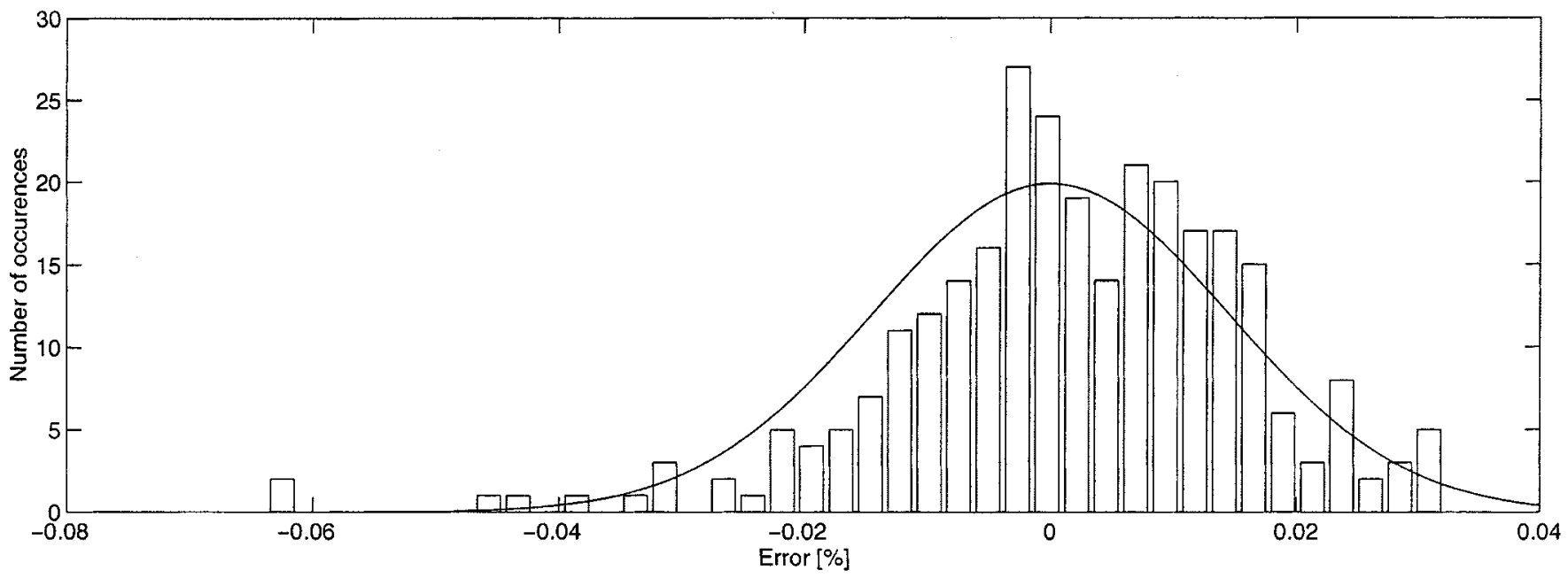

Fig. 2. Results from the heats between January-April 2001.

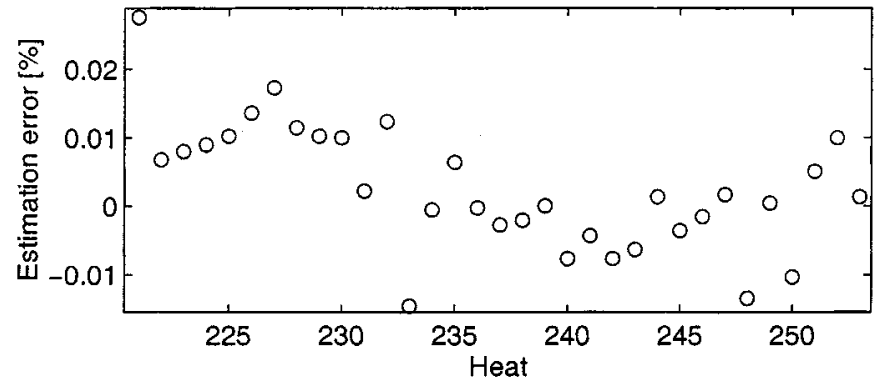

Fig. 3. Estimation error over time.

\section{A. Heat Operators}

From Fig. 4 (lower plot), it is evident that there are differences in the quality of the estimate, depending on which operator team runs the converter process.

There appears to be a negative correlation between how well the final carbon content is achieved by the operators (upper plot of Fig. 4) and how well the algorithm performs. This is, however, dependent upon the values of the observer parameters. The main conclusion is that there seems to be some difference in procedure between the teams, which is reflected in different behavior of the process.

The number of blows for each team is between 30-100 in the data for Fig. 4 and, thus, the difference between the teams is assumed to be statistically guaranteed. Differences between individual operators cannot be determined due to insufficient data.

\section{B. Bath Level}

Possible causes of the estimation error are variations in the distance between the lance and the metal surface, caused by variations in the bath level, i.e., the level of the liquid metal surface. This distance affects the impact area of the oxygen jet from the lance and, thus, has a direct influence on the decarburization rate. Since the growth of foaming slag is also dependent on this
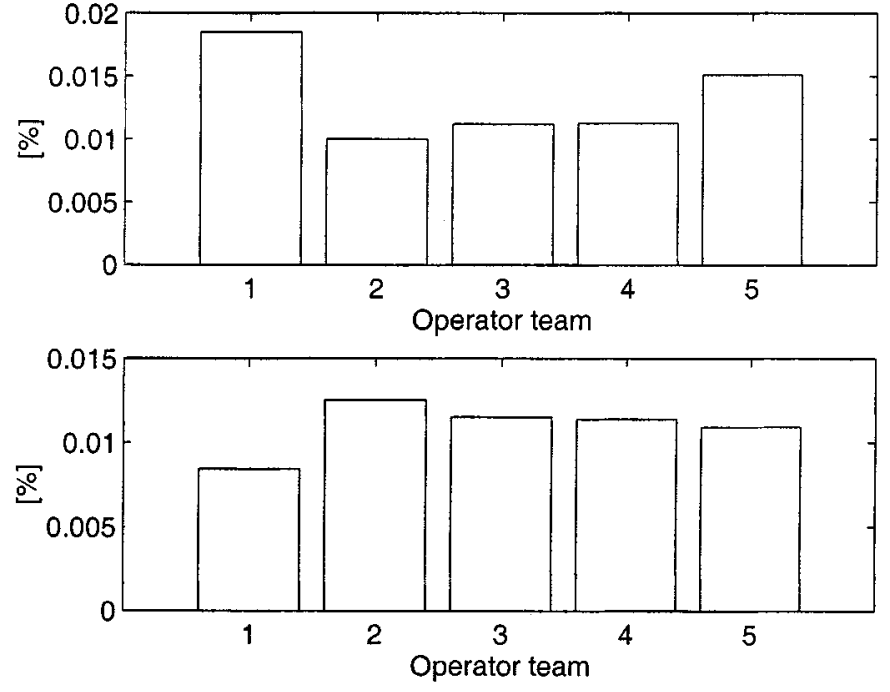

Fig. 4. Difference between operator teams. Upper plot shows mean error in achieved carbon content, while lower plot shows mean estimation error of the observer.

distance, and the foam volume is important for the decarburization process, there is also an indirect influence from the bath level on the decarburization rate.

Fig. 5 shows measurements of the bath level using an oxygen probe. This measurement is regarded as unreliable and noisy, but its filtered counterpart shows that the signal indeed carries information about the bath level. This can be seen by noting that the bath level seems to have fallen $20 \mathrm{~cm}$ during 500 heats, which is roughly the expected value due to wear of the converter lining.

The operator makes an estimate of the bath level, based upon a number of factors, including measurements of the composition of the slag. This estimate, shown in Fig. 5 as "manually set bath level," is used as the true bath level, when calculating the absolute lance height, to obtain a certain distance between lance and metal surface. It is not clear how reliable this estimate is and, from the figure, it is evident that it does not show much 


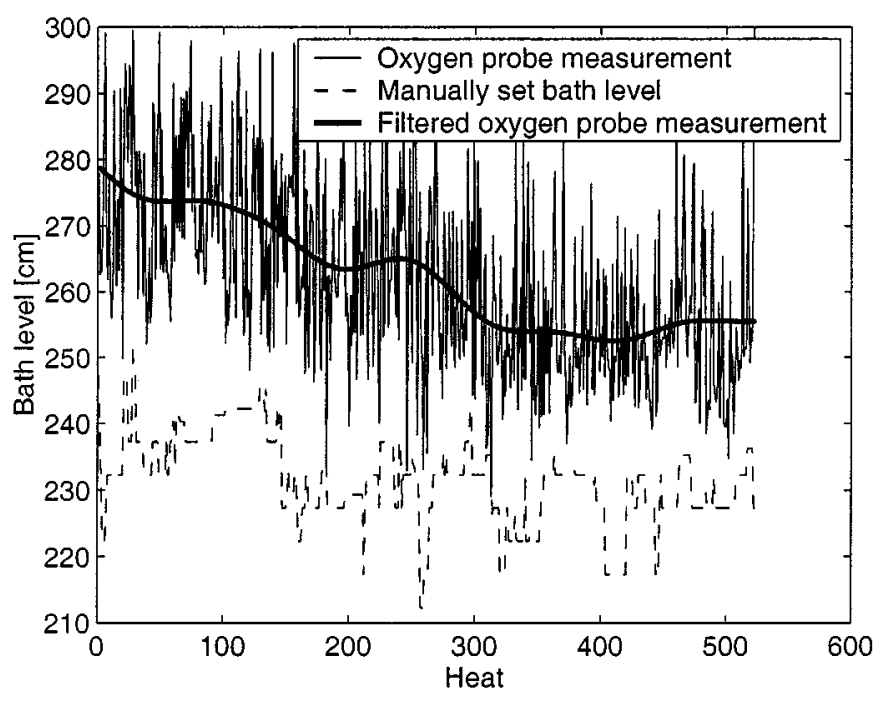

Fig. 5. Variations in bath level.

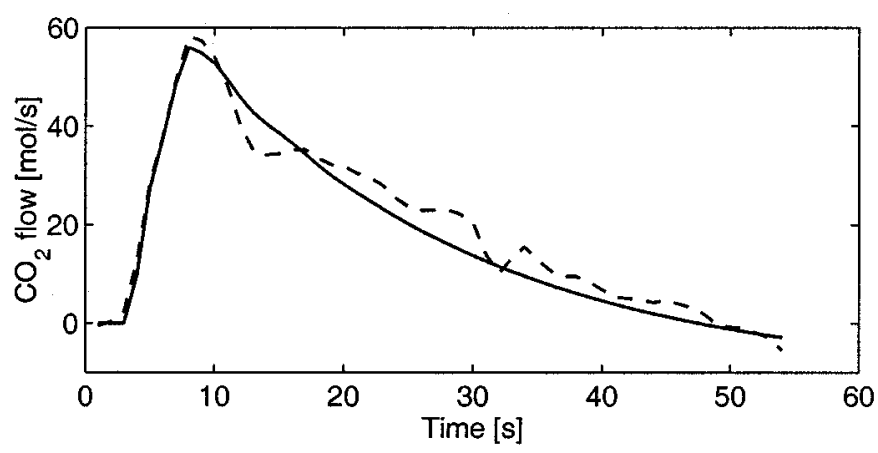

Fig. 6. Response in off-gas flow of $\mathrm{CO}_{2}$ when adding $600 \mathrm{~kg}$ of dolomitic lime. Dashed line shows the measured response while the solid line is the simulated response of a fourth-order identified linear model.

similarity to the bath level measurement, apart from the overall trend.

\section{FUTURE WORK}

Further research is required on how to deal with the effects of additives, in particular, dolomitic lime, but also ferrosilicon.

When adding dolomitic lime, it will melt and release $\mathrm{CO}_{2}$ to the liquid metal. A model for this process can be obtained by measuring the excess $\mathrm{CO}_{2}$ in the off-gases after the additive is released. Fig. 6 shows measured and simulated response in $\mathrm{CO}_{2}$ flow from a $600-\mathrm{kg}$ addition of dolomitic lime.

The existing strategy for dealing with additions of dolomitic lime is to subtract the simulated flow of carbon $y_{D}$ due to the additive from the measured carbon flow from the converter $y$ and obtaining the decarburization rate $y_{0}$ from this difference [4]. Fig. 7 shows the principal structure of this approach, applied to the method used in this paper. Adding of dolomitic lime is represented by the input $w_{D}$ and the estimated carbon flow in the off-gases resulting from this additive is denoted $\hat{y}_{D}$. Estimated carbon flow in the off-gases due to decarburization is denoted $\hat{y}_{0}$, while $u$ and $w$ are input signals to the converter process.

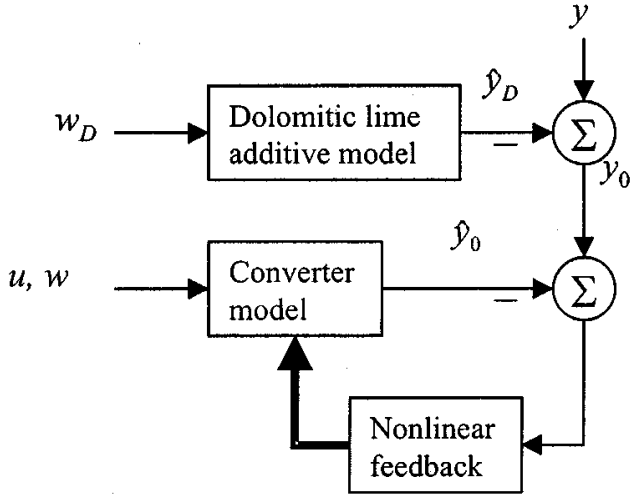

Fig. 7. Static compensation for the effects of dolomitic lime additives.

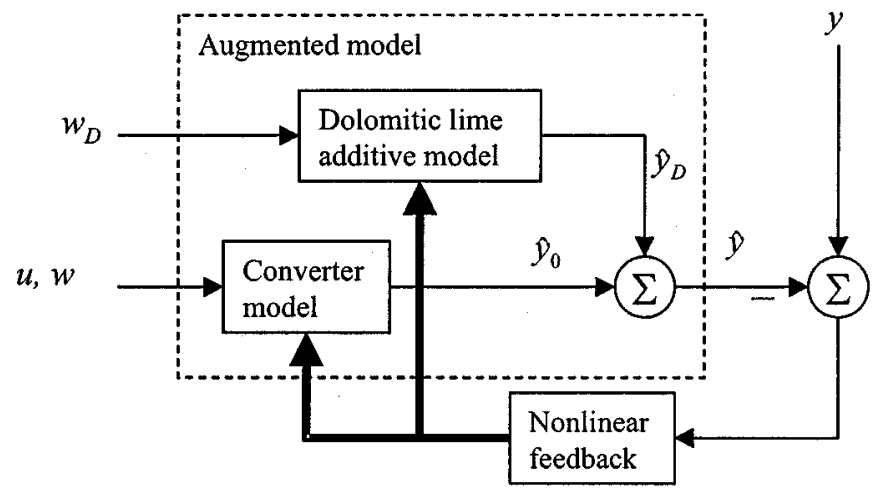

Fig. 8. Dynamic compensation for the effects of dolomitic lime additives with feedback of the output error into the dolomitic lime model.

There are, however, large uncertainties in the model of the addition of dolomitic lime and, therefore, this static method will create disturbances in the decarburization rate fed back to the converter model.

One way of dealing with this problem is to include the model for the dolomitic lime reaction with the converter model and apply feedback to this augmented model (Fig. 8). In this context, it could also be advantageous to utilize the redundant information in the off-gas analysis to be able to separate the contribution from decarburization from that of dolomitic lime addition into the carbon flow in the off-gas.

\section{REFERENCES}

[1] A. Johansson, A. Medvedev, and D. Widlund, "Model-based estimation of decarburization rate and carbon content in the basic oxygen steelmaking process," in Conf. Rec. IEEE-IAS Annu. Meeting, Oct. 2000, CD-ROM.

[2] D. Widlund, A. Medvedev, and R. Gyllenram, "Toward model-based closed-loop control of the basic oxygen steelmaking process," in Preprints 9th IFAC Symp. Automation in Mining, Mineral and Metal Processing, 1998.

[3] MEFCON. [Online]. Available: http://www.mefos.se/mefcon.htm.

[4] D. Bergman and P. Hahlin, "Experience of waste gas analysis based control system for the LD-LBE-process at SSAB Tunnplåt AB, Luleå, Sweden," presented at the 2nd European Oxygen Steelmaking Congr. (EOSC '97), Taranto, Italy, Oct. 1997.

[5] A. Johansson, A. Medvedev, and D. Widlund, "Model-based estimation of metal analysis in steel converters," in Proc. 39th IEEE Conf. Decision and Control, Dec. 2000, pp. 2017-2022. 


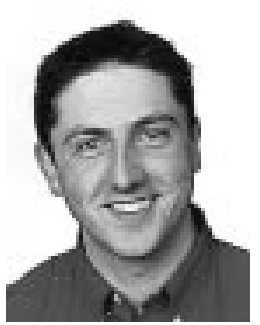

Wolfgang Birk was born in Merzig, Germany, in 1968. He received the M.S. degree in electrical engineering from Saarland University, Saarbrücken, Germany, in 1997. He is currently working toward the Ph.D. degree in the Control Engineering Group, Luleå University of Technology, Luleå, Sweden.

His research interests are interaction in multivariable control systems and soft sensors.

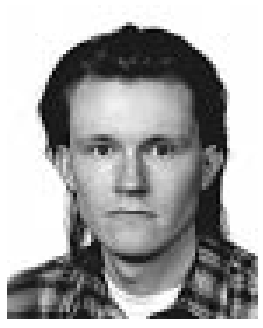

Andreas Johansson was born in Luleå, Sweden, in 1972. He received the M.Sc. degree in computer science in 1997 from Luleå University of Technology, Luleå, Sweden, where he is currently working toward the Ph.D. degree in the Control Engineering Group.

His primary research interests are fault detection and estimation in nonlinear systems.

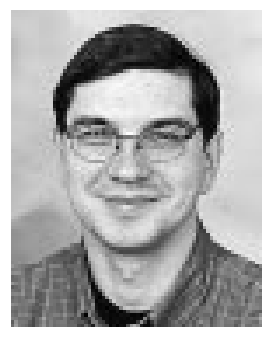

Alexander Medvedev was born in Leningrad, U.S.S.R., in 1958, He received the M.Sc. (honors) and $\mathrm{Ph} . \mathrm{D}$. degrees in control engineering and the Docent degree from Leningrad Electrical Engineering Institute (LEEI), Leningrad (St. Petersburg), U.S.S.R., in 1981, 1987, and 1991, respectively.

From 1981 to 1991, he subsequently held positions as a System Programmer, Assistant Professor, and Associate Professor in the Department of Automation and Control, LEEI. He was with the Process Control Laboratory, Åbo Akademi, Finland, during a year-long research visit in 1990-1991. In 1991, he joined the Computer Science and System Engineering Department, Luleå University of Technology, Luleå, Sweden, as an Associate Professor in the Control Engineering Group (CEG). In February 1996, he was promoted to Docent. From October 1996 to December 1997, he served as Acting Professor of Automatic Control in the CEG, where, in January 1998, he was elected Full Professor of Automatic Control. Since October 2001, he has also been a Professor of Automatic Control at Uppsala University, Uppsala, Sweden. He is presently involved in research on fault detection, time-delay systems, and alternative parameterization methods in analysis and design of dynamic systems. He has authored or coauthored more than 90 scientific papers. Since 1997, he has supervised activities within the Center for Process and System Automation (ProSA) at Luleå University of Technology.

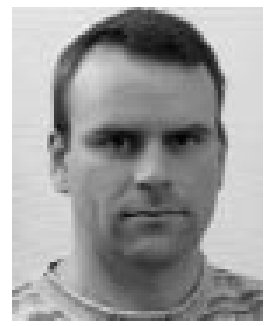

Robert Johansson was born in Luleå, Sweden, in 1965. He received the M.Sc. degree in automatic control from Luleå University of Technology, Luleå, Sweden, in 1999.

Since 1987, he has been with SSAB Tunnplåt AB, Luleå, Sweden, where he is currently an R\&D Engineer in the IT Department. 